\title{
SUBCONJUNCTIVAL DIROFILARIA REPENS
}

\author{
I. Neichev ${ }^{1}$, K. Slaveykov ${ }^{1}$, K. Trifonova ${ }^{1}$ and B. Chakarova ${ }^{2}$ \\ ${ }^{1}$ Department of Ophthalmology and General Medicine, Medical Faculty, Trakia University, \\ Stara Zagora, Bulgaria \\ 2Department of Parasitology, Medical Faculty, Trakia University, Stara Zagora, Bulgaria
}

Summary. Dirofilariasis is a relatively rare zoonotic disease that affects mainly dogs. The two most common representatives are Dirofilaria immitis and Dirofilaria nochtiella. Studies have documented the spread of the disease across Central and Eastern Europe in recent years due to global changes. In Bulgaria since 2001 there are around 20 cases of dirofilariasis in dogs registered yearly. We report a case of subconjuctival dirofilariasis in a 47-year-old woman living in Southwestern Bulgaria. The infection manifested as a painful, inflamed nodule in the left eye, containing a vital parasite. Morphological analysis aroused suspicion for a filarial worm belonging to the genus Dirofilaria. Subsequent analysis confirmed suspicions and identified the parasite as Dirofilaria repens. After removal, the subconjunctival nodule completely resolved and the patient was successfully cured.

Key words: ophthalmology, dirofilariasis

\section{INTRODUCTION}

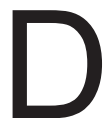
irofilaria is a genus of the family Onchocercidae, order Spirurida, subtype Nematoda. Heartworms can be divided into two main groups: Dirofilaria immitis and Dirofilaria nochtiella. The former is found mainly in the heart and lungs of dogs and the latter subcutaneously in domestic and wild carnivores (dogs, cats, foxes) [1]. Dirofilaria repens, which affects people, is a member of Dirofilaria nochtiella. Dirofilariasis is a zoonotic disease that rarely affects humans. Infection occurs by blood-sucking arthropods - mosquitoes, ticks, fleas. They are infected with microfilariae by sucking from infected animals, which after maturation are spread to other vertebrates.

Mature heartworms prefer tissues and organs of vertebrates, while the immature forms prefer blood and lymph nodes. Dirofilaria repens is most often found as a subcutaneous parasite in dogs [2]. 
Since the human body is not a suitable host for heartworms, the subconjunctivial form of the worm does not mature [3].

The disease is most widespread in countries in Eastern Europe, the Middle East, Asia and Africa [4].

The first case of dirofilariasis was reported by Angelo Pace in 1867 in Palermo. Studies show that Dirofilaria repens is the cause of heartworm disease in humans in 42.3\% of the cases in Italy, $13.3 \%$ in France, $7.8 \%$ in Sri Lanka and $4.3 \%$ in Turkey [5].

In humans, the parasite is most commonly found in areas of the head, chest and upper limbs, situated most often subcutaneously and rarely internally. In our country cases of heartworm disease are mainly registered in the eyelids, the last case was reported by Asoc. Prof. Marin Atanasov Ph.D. in 2004 in Plovdiv. Subconjunctival localization is very rare [6].

Proper diagnosis is made histologically by identifying the nematode and morphological characteristics distinguishing it from other heartworms. The identification of the parasite is performed based on the macroscopic and microscopic characteristics evaluation. Hematoxylin-Eosin and PAS staining was used [7].

The only effective treatment of subconjunctival heartworm disease is surgical removal of the parasite. Mosquito population must be controlled and infected dogs need to be treated. Other protection methods are not known [8].

\section{RESULTS AND DISCUSSION}

We present a case of a 47-year-old woman from Stara Zagora, Bulgaria. The patient was admitted in the clinic for the first time. She complained about a sudden pain in the right eye, itching, swelling, redness and a feeling of movement in the eye. A migratory subcutaneous swelling of the skin accompanied by itching for at least one year was reported. Epidemiological data suggested she has not visited endemic areas abroad and has not had contacts with pets.

Corrected visual acuity was 1.0. During slit lamp examination a well defined cystic formation was found in the temporal part of the left eyeball. The cyst was $3 \mathrm{~mm}$ from the limbus and had a mobile nematode in it. No other abnormalities in ophthalmic status of the left eye were found (Fig. 1).

After incision of the cystic formation from the lower fornix dense synechiae were found between the conjunctiva and Tenon's capsule. After blunt dissection of the tissue had been done, a vital, threadlike nematode was extracted. Laboratory tests reported eosinophilia. The parasite was sent to parasitological laboratory where it was identified as a member of Nemathelminthes (Dirofilaria repens) with a length of about $10 \mathrm{~cm}$ (Fig. 2).

The forward rounded end was wider than the rear, and it was established microscopically that the parasite had a non-segmented cuticle. Based on the macroscopic and microscopic characteristics the worm was identified as immature form of $D$. 
repens (Fig. 3). Subconjunctival localization and epidemiological data support this diagnosis. After surgical removal of the nematode the patient was treated with Zentel (Albendazole) $400 \mathrm{mg} 10 \mathrm{mg} / \mathrm{kg}$ for 10 days.

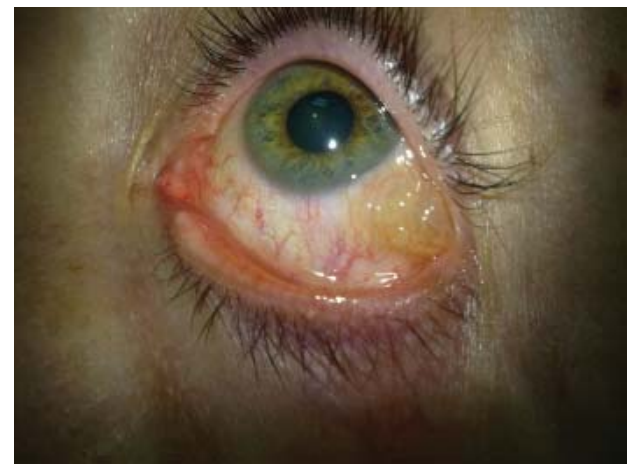

Fig. 1. During slit lamp examination a well defined cystic formation was found in the temporal part of the left eyeball. The cyst was $3 \mathrm{~mm}$ from the limbus and had a mobile nematode in it. No other abnormalities in ophthalmic status of the left eye were found

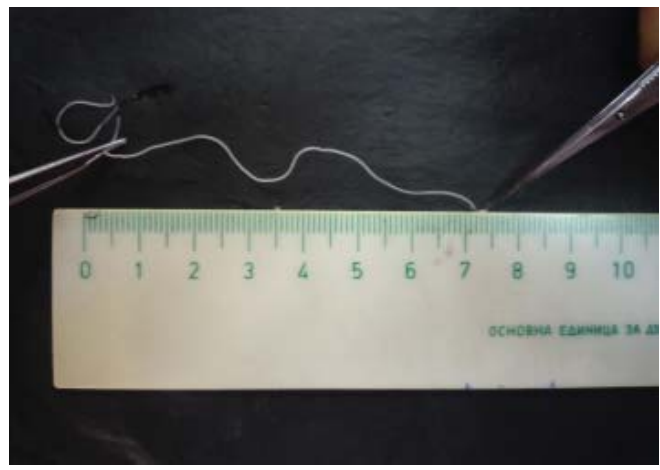

Fig. 2. The parasite was sent to parasitological laboratory where it was identified as a member of Nemathelminthes (Dirofilaria repens) with a length of about $10 \mathrm{~cm}$

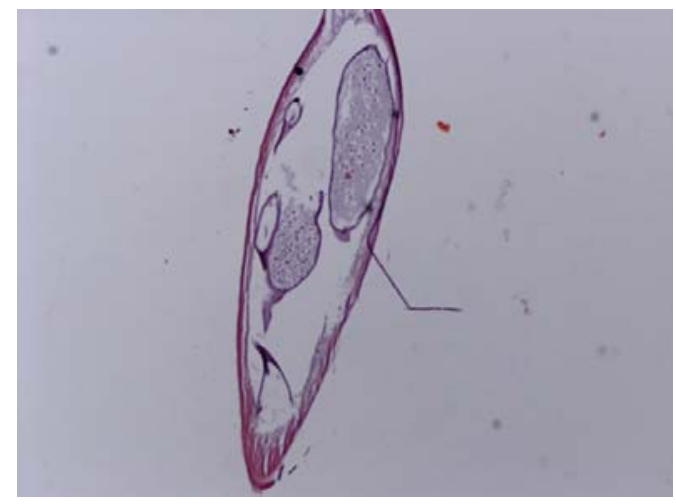

Fig. 3. Based on the macroscopic and microscopic characteristics the worm was identified as immature form of D. repens

\section{CONCLUSIONS}

Data on people infected with Dirofilaria repens with subconjunctival location is scarce. As the source of infection are pets, their treatment plays a major role in limiting the spread of the parasite. In recent years the case of heartworm disease have been documented mainly in Mediterranean countries - Greece, Italy, Spain, France, Israel $[9,10]$. In Bulgaria since 2001 there have been about 20 cases of 
affected dogs annually. There is no medication in Bulgaria for treating the animals at present time, but prevention is possible by using Ivermectin once a month during the summer when there are mosquitoes. Blood smear is required for the diagnosis of the disease as well as serology - establishing antibody and PCR for DNA from microfilariae in mosquitoes.

Although it is a rare disease, dirofilariosis must be considered in epidemiological and parasitological studies. Because of the wide variety of locations of the worm and symptoms associated with them, dirofilariosis becomes interdisciplinary problem involving veterinarians, ophthalmologists, dermatologists and general practitioners.

\section{REFERENCES:}

1. Arvanitis, P. et al. Ophthalmic dirofilariasis. - Am. J. Ophthal.,123, 1997, 689-691

2. Fu entes, I. et al. Human subcutaneous dirofilariosis caused by Dirofilaria repens in Ibiza, Spain. - Am. J. Trop. Med. Hyg., 51, 1994, 401-404

3. G a u t a m, V. et al. Subconjunctival infection with Dirofilaria repens. - Jpn. J. Infect. Dis., 55, 2002, № 2, 47-48.

4. Marty, P. 1997, Human dirofilariasis due to Dirofilaria repens in France. A review of reported cases. - Parassitologia, 39, 1997, № 4, 383-386.

5. Pamplione, S., T. Canestri et F. Rivasi. Human dirofilariosis due to Dirofilaria (Nochtiella) repens: a review of world literature. - Parasitologia, 37, 1995, 149-193

6. Rou h e t t e, H. et al. Ocular filariasis: not strictly tropical. - Ophthalmologica, 213, 1999, 206-208.

7. Soner, İ., Ö. Kadri et D. Nizami, Subconjcuntival infention with Dirofilaria repens. - Annals of Saudi Medicine, 22, 2002, № 1-2.

8. Soylu, M. 1993, Dirofilariasis: an uncommon parasitosis of the eye. - Br. J. Ophthal., 77, 1993, 602-603

9. J elin e k, T., J. Schulte-Hillen et T. Loscher. Human Dirofilariasis. - Int. J. Dermatol., 35, 1996, № $12,872-875$.

10. Vakalis, N. et al. 1999, Improved detection of Dirofilaria repens DNA by direct polymerase chain reaction. - Parasitol. Int., 48, 1999, 145-150.

Corresponding author:

Kiril Slaveykov, MD

Department of Ophthalmology and General Medicine

Medical Faculty

Trakia University

Bg - 6000 Stara Zagora

(ग) +359886712078

e-mail: kirilslaveykov@gmail.com 